\title{
Maternal distress in the context of their child's type 1 diabetes: Exploring the role of adaptive maternal emotion regulation on child outcomes.
}

Cynthia Van Gampelaere ${ }^{1}$, Tine Vervoort ${ }^{1}$, Koen Luyckx ${ }^{2}$, Annick De Paepe ${ }^{1}$, Sara Van Aken ${ }^{3}$, Liesbet Goubert ${ }^{1}$

Cynthia Van Gampelaere, Department of Experimental, Clinical and Health Psychology, Ghent University, Henri Dunantlaan 2, 9000 Ghent, Belgium

ORCHID iD:0000-0001-9667-2124

email: cynthia.vangampelaere@ugent.be

Phone: +3292648650

Tine Vervoort, Department of Experimental, Clinical and Health Psychology, Ghent University, Henri Dunantlaan 2, 9000 Ghent, Belgium

email: tine.vervoort@ugent.be

Phone: +32926464 71

Koen Luyckx, Department of School Psychology and Development in Context, University of Leuven, Tiensestraat 102 (3717), 3000 Leuven, Belgium

email:koen.luyckx@kuleuven.be

Phone: +3216325978

Annick De Paepe, Department of Experimental, Clinical and Health Psychology, Ghent University, Henri Dunantlaan 2, 9000 Ghent, Belgium

ORCHID iD: 0000-0002-9366-3692

email: annick.depaepe@ugent.be

Phone: +3292646392

Sara Van Aken, Department of Pediatrics, University Hospital Ghent, De Pintelaan 185, 9000 Ghent, Belgium

email:sara.vanaken@ugent.be

Phone: +3293322430

Liesbet Goubert, Department of Experimental, Clinical and Health Psychology, Ghent University, Henri Dunantlaan 2, 9000 Ghent, Belgium

ORCHID iD: 0000-0002-7657-5148

email:liesbet.goubert@ugent.be

Phone: +3292646262

Address correspondence to Cynthia Van Gampelaere

Department of Experimental-Clinical and Health Psychology, Ghent University, Henri

Dunantlaan 2 B- 9000 Ghent, Belgium

Tel: +32 (0)9 2649108

Email: Cynthia.VanGampelaere@UGent.be 


\section{Maternal distress in the context of their child's type 1 diabetes:}

\section{Exploring the role of adaptive maternal emotion regulation on child}

\section{outcomes.}

Parents of children with Type 1 diabetes (T1D) experience high levels of distress, which may negatively impact child functioning. However, little is known about mechanisms that may buffer the adverse impact of parental distress. The current study explored the possible buffering role of maternal adaptive cognitive emotion regulation (CER) for the relationship between maternal distress and child psychological functioning. Forty-three children with T1D (8-15 years) completed measures assessing trait anxiety and depressive symptoms. Their mothers reported on general distress, illness-related parenting stress, and adaptive CER. Maternal illness-related parenting stress (but not general distress) was significantly associated with child psychological functioning. No buffering role for maternal adaptive CER was observed. As the current study is rather preliminary, future research using other methods to examine maternal adaptive CER, and examining other parental variables that may buffer against the negative impact of parental distress is warranted.

Keywords: pediatric psychology; diabetes Mellitus type 1; parental distress; cognitive emotion regulation

\section{Introduction}

Type 1 diabetes (T1D) brings along many challenges in the lives of children, as they have to follow a complex and lifelong treatment regimen. They are at risk of developing anxiety, mood, and eating disorders (Butwicka et al., 2016; Geisbüsch \& Bühren, 2015). Not only the child is affected; T1D is a family disease (Williams, Laffel, \& Hood, 2009), and parental reactions of stress (Cline, Schwartz, Axelrad, \& Anderson, 2011), anxiety, and depression are common after diagnosis (Streisand et al., 2008). While most parents adapt well over time 
(Lowes, Gregory, \& Lyne, 2005), significant levels of distress (i.e., anxiety, depression, and parenting stress) may remain several years later (Whittemore, 2012).

Parental distress may contribute to worse parental functioning (Helgeson, Becker, Escobar, \& Siminerio, 2012), but also impact the child with diabetes (Whittemore, 2012). Parental distress has been associated with problematic child behaviors (Hilliard, Monaghan, Cogen, \& Streisand, 2011), child depressive symptoms (Jaser, Whittemore, Ambrosino, Lindemann, \& Grey, 2007), reduced child quality of life (Nieuwesteeg et al., 2016), school absenteeism, and poor child glycemic control (Cameron, Young, \& Wiebe, 2007; Haugstvedt, Wentzel-Larsen, Graue, Søvik, \& Rokne, 2010). Importantly, parental distress can be situated on different levels, with each level having differential effects. A five-year study showed that parental general distress predicted a decrease in glycemic control, while parental diabetesspecific distress predicted an increase in child depressive symptoms but also improvements in glycemic control (Helgeson et al., 2012). As a result of the rising incidence of T1D (Patterson et al., 2012), a growing number of parents is confronted with pediatric T1D. Hence, understanding factors that may counteract the negative effects of parental distress upon children with T1D is crucial.

Drawing upon resilience literature, focusing on strengths of patients and their families to achieve the best possible outcomes in the face of diabetes is of paramount importance (Hilliard, McQuaid, Nabors, \& Hood, 2014). Parental coping has been suggested to be particularly relevant in this regard (Jaser, Linsky, \& Grey, 2014). Cognitive emotion regulation (CER) is a specific type of coping, which refers to the cognitive way of managing emotionally arousing information (Garnefski, Kraaij, \& Spinhoven, 2001). Different adaptive strategies can be used to regulate negative emotions, such as acceptance, refocus on planning, positive refocus, positive reappraisal, and putting into perspective. Positive reappraisal (Glidden, Billings, \& Jobe, 2006; van der Veek, Kraaij, \& Garnefski, 2009) and acceptance 
(Norizan \& Shamsuddin, 2010) have been associated with less distress and more wellbeing in parents of children with developmental disabilities. In mothers of adolescents with T1D, acceptance was related to less maternal anxiety and depression (Jaser et al., 2014). Research in young adults with chronic conditions, including T1D, supports the idea that adaptive emotion regulation strategies (positive refocus, positive reappraisal, and putting into perspective) are associated with less distress (Kraaij \& Garnefski, 2012, 2015). Further, the use of adaptive strategies might play a buffering role as shown in a recent study where the association between dysfunctional attitudes and distress was weaker in students reporting more adaptive CER (Vanderhasselt et al., 2014). However, whether parental adaptive CER strategies buffer parental distress from exerting its negative impact upon the child with T1D has yet to be examined.

Our first aim was to extend previous findings regarding associations between parental distress and child psychological functioning. In line with previous research, we hypothesize that both maternal general and illness-related distress will contribute to more depressive and anxiety symptoms in children with T1D. Our second aim was to explore the buffering role of maternal adaptive CER. We hypothesize that the relationships between maternal distress and maladaptive child outcomes will be less pronounced for mothers reporting more adaptive CER.

\section{Method}

\section{Participants}

Participants, recruited from Ghent University Hospital, were part of a larger study consisting of multiple study aims. Findings pertaining to other study aims have been reported elsewhere (see Vervoort et al., 2011). To be eligible for participation, the child (a) had to be diagnosed with T1D, (b) aged 8-15 years, (c) was not suffering another physical or pervasive developmental disorder, and (d) parent and child had to be Dutch-speaking. Of 74 families 
who were approached, 49 entered the study (49 children, 44 mothers and 16 fathers); 18 refused participation (mostly because of lack of time) and 7 did not take part due to rescheduled pediatrician appointments. Due to the small number of participating fathers, only mother-child data were used. After removal of one case with too much missing data, the final sample consisted of 43 mother-child dyads. Demographics are presented in Table 1.

- Insert Table 1 about here -

\section{Procedure}

All families with children with T1D being treated in Ghent University Hospital at the moment of inclusion were sent an invitation letter. Upon agreement to participate, they were contacted and an appointment was made prior to their next hospital consultation. The first part of the study assessed parent and child characteristics by means of self-report questionnaires. Only data of this part were used in the current study. For details of the second part, see Vervoort et al. (2011). In the hospital the research assistant obtained written parent consent and child assent. Subsequently, self-report measures were completed by children and parents. Participants did not receive any compensation for participating. The study was approved by the Ethical Committee of Ghent University Hospital.

\section{Measures}

Parent measures

Maternal General Distress. The Dutch Hospital Anxiety and Depression Scale (HADS) (Spinhoven et al. 1997; Zigmond \& Snaith, 1983) is a 14-item self-report scale measuring anxious and depressive symptoms during the past week on a 4-point Likert scale. The total score, with a range of 0 to 42 , is computed by summing the anxiety and depression subscale scores. The Dutch version has shown good internal consistency (Spinhoven et al., 1997). Cronbach's alpha in the current study was .92 . 
Illness-related Parenting Stress. The Dutch Pediatric Inventory for Parents (PIP) (Vrijmoet-Wiersma et al., 2010) examines parental stress related to caring for a child with a medical condition (Streisand, Swift, Wickmark, Chen, \& Holmes, 2005). Parents are asked to indicate the frequency and perceived difficulty of 42 stressful events on two 5-point Likert scales $(1=$ never/ not at all, $5=$ very often/ extremely $)$. The total frequency and difficulty scales correlated strongly in the current study $(r=.86, \mathrm{p}<.001)$; therefore it was decided to use the composite score (ranging from 42 to 210) in subsequent analyses. Internal consistency was excellent $(\alpha=.98)$.

Maternal Adaptive Cognitive Emotion Regulation. The Dutch Cognitive Emotion Regulation Questionnaire (CERQ) is a 36-item questionnaire measuring nine cognitive emotion regulation strategies (Garnefski et al., 2001) of which five are considered adaptive CER and hence used in the present study; acceptance, refocus on planning, positive refocusing, positive reappraisal and putting into perspective. Mothers were asked to indicate on a 5-point Likert scale how often they think in a certain way when they experience something annoying or bad $(1=($ almost $)$ never, $5=($ almost $)$ always $)$. Following previous studies (e.g., Hoorelbeke, Marchetti, De Schryver, \& Koster, 2016), adaptive emotion regulation was indexed by calculating the mean (ranging from 4 to 20) of these 5 subscales, showing adequate internal consistency $(\alpha=.88)$.

\section{Child measures}

Child anxiety. The 20-item Trait version of the State-Trait Anxiety Inventory for Children (STAIC-trait) (Spielberger, Edwards, Lushene, Montuori, \& Platzek, 1973) taps into children's anxious disposition by asking them, using a 3-point Likert scale $(0=$ almost never, $2=$ often), how they generally feel. Scores range from 0 to 40 . The Dutch version (Bakker, Van Wieringen, van der Ploeg, \& Spielberger, 1989) indicated good reliability $(\alpha=.81)$. 
Child depression. Child depressive symptoms were assessed using the Dutch Children's Depression Inventory (CDI) (Kovacs, 1981; Roelofs et al., 2010), consisting of 27 items scored from 0 to 2 . Children are requested to choose one out of three descriptions, that best describes how they have been feeling/thinking the past two weeks. Scores range from 0 to 54. The CDI has shown good internal consistency in children with T1D (e.g., Jaser et al., 2014). Cronbach's alpha in the current study was .63.

\section{Statistical analysis}

Statistical analyses were conducted using SPSS 23.0. Pearson correlations were examined. To determine a parsimonious model for child functioning, a multiple regression model was estimated for each dependent variable (CDI and STAI-trait), with both maternal distress variables (HADS and PIP) as predictors. Each model controlled for child age, but not for child gender and time since diagnosis, since these were not correlated with the predictors or outcomes (Table 2). Both models were reduced stepwise (method backward). In each step, the least significant predictor was eliminated if its two-tailed $p$-value was larger than .10. Normality was checked by visually inspecting the histograms and QQ-plots of the residuals. Multicollinearity of the predictors and absence of outliers and influential cases were also investigated and effect sizes $r$ were calculated (Durlak, 2009). To examine whether maternal adaptive CER served as a buffer, two multiple regression models were examined for each dependent variable (method enter). In each model, the interaction between adaptive CER and one of the two predictors (HADS and PIP) was added, whilst controlling for child gender, and the main effects of maternal adaptive CER and the respective maternal distress variable. All continuous independent variables were standardized. 


\section{Results \\ Correlation analyses}

Pearson correlations are reported in Table 2. Both maternal distress variables were strongly interrelated $(r=.72)$, yet not so much as to imply multicollinearity (VIF $\leq 2.71)$. Similarly, both child outcomes showed a strong interrelation $(r=.66)$. Both maternal general distress and maternal illness-related parenting stress correlated significantly with child depressive symptoms, but not with anxiety. Adaptive CER showed no significant correlations with maternal distress and with child outcomes ( $r$ 's $\leq .11, \mathrm{~ns})$.

- Insert Table 2 about here -

\section{The role of maternal distress in explaining child outcomes}

The two final multiple regression models were statistically significant (see Table 3 ). After stepwise elimination of non-significant predictors, the remaining predictors accounted for respectively $25 \%$ and $12 \%$ of the variance in child anxiety and depressive symptoms. Girls experienced higher levels of anxiety but not more depressive symptoms. In contrast to our hypotheses, maternal general distress showed no unique predictive value in explaining child outcomes and was removed from the final models. As expected, maternal illness-related parenting stress was a significant predictor of child anxiety and depressive symptoms.

- Insert Table 3 about here -

\section{Moderation by maternal adaptive cognitive emotion regulation}

Two out of four estimated regression models were found to be significant. The model including illness-related parenting stress $(F=3.26, p=.02)$ explained $26 \%$ of the total variance in child anxiety, whereas the predictors of the model including general distress $(F=$ $2.97, p=.03$ ) accounted for $24 \%$ of the variance in child anxiety. None of the models generated main effects of maternal adaptive CER upon the child outcomes $(\beta$ 's $<.15, p$ 's $>$ 
.37). Counter to expectations, results showed no significant moderation effects on the associations of maternal general distress and illness-related parenting stress with child anxiety and depression $(\beta$ 's $<.18, p$ 's $>.27)$.

\section{Discussion}

The present multi-informant dyadic study showed that mothers with higher levels of illnessrelated parenting stress had children who reported more anxiety and depressive symptoms. In contrast to expectations, maternal adaptive cognitive emotion regulation (CER) did not buffer against the negative influence of maternal distress upon child functioning.

Our results confirmed and extended previous findings regarding intergenerational associations in childhood T1D. In line with previous research (Mullins et al., 2004), mothers experiencing more illness-related parenting stress had children with more depressive symptoms. Child anxiety - although common in children with T1D (Kovacs, Goldstone, Obrosky, \& Bonare, 1997) - is often not included when child outcomes are examined. Given that parental fears/stress may pass on to their child through observational learning (Aktar, Majdandzic, De Vente, \& Bogels, 2014), the finding that children with T1D experience more anxiety when their mothers report more illness-related parenting stress is not surprising. Further, in line with affective-motivational theories (e.g., Goubert \& Simons, 2013), mothers who experience illness-related parenting stress may be more protective of their child to avoid stressful/feared events, such as hypoglycemia. Both the observation of distress in their parents as well as the effect of protective behaviors themselves (e.g., keeping child home from social activities) may induce child anxiety and depressive symptoms (Chow, Otis, \& Simons, 2016). Future research is warranted focusing on such intervening mechanisms.

Maternal general distress showed no unique predictive value towards child outcomes. This is in contrast to earlier research of Helgeson et al. (2012) who found that general distress 
was related to child depression, and diabetes-specific distress was not, although it predicted child depression over time. Other studies in the context of childhood T1D that only tapped into parental general distress did also find associations with maladaptive child outcomes (Cameron et al., 2007; Hilliard et al., 2011; Jaser et al., 2007). A possible explanation for the current finding might be that stress related to the child's diabetes is a more proximally related to child (psychological) functioning and more likely to be communicated towards the child (Sieberg, Williams, \& Simons, 2011).

The present study was the first to examine interpersonal effects of CER in the context of pediatric T1D. In contrast to hypotheses, results revealed no moderation effects of maternal adaptive CER on the associations between maternal distress and child outcomes. Possibly, these effects remained undetected due to the small sample size. An alternative explanation might be that maternal adaptive CER stimulates positive child outcomes, such as child positive emotions and treatment adherence, instead of buffering negative outcomes. Future research should examine this possible explanation.

The current study has some limitations. First, the use of a cross-sectional design prevented assessing the plausible bidirectionality of the effects. Second, the small sample consisted of only mothers and children above 8 years of age. This made it impossible to examine differences between mothers and fathers, and explore hypotheses in younger children with T1D where parental distress is even more common (Patton, Dolan, Henry, \& Powers, 2008; Patton, Dolan, Smith, Thomas, \& Powers, 2011). A final limitation is the use of selfreport measures. Future longitudinal research should use other methods to examine parental adaptive CER (e.g., observation), in larger samples, preferably including both parents.

In conclusion, our preliminary findings highlight the associations between maternal illness-related parenting stress and maladaptive child outcomes in childhood T1D, pointing to the importance of screening for parenting stress in the clinic. Given those significant 
relationships, future research should use other methods to examine adaptive CER, examine other possible moderators that may have a buffering role, and develop an encompassing model of parental influence in the context of pediatric T1D.

Acknowledgments, the authors would like to thank Veerle Hiel and Katrien Geerinck for their help in this study.

Cynthia Van Gampelaere is a PhD student of the Fund for Scientific Research - Flanders (FWO, Belgium).

Conflicts of Interest, the authors declare that they have no conflicts of interest.

Ethical approval, all procedures followed were in accordance with the ethical standards of the responsible committee on human experimentation of Ghent University Hospital and with the 1964 Helsinki declaration, as revised in 2000.

Informed consent was obtained from all individual participants included in the study. 


\section{References}

Aktar, E., Majdandzic, M., De Vente, W., \& Bogels, S. M. (2014). Parental social anxiety disorder prospectively predicts toddlers' fear/avoidance in a social referencing paradigm. Journal of Child Psychology and Psychiatry and Allied Disciplines, 55(1), 77-87.

Bakker, F. C., Van Wieringen, P. C. W., van der Ploeg, H. M., \& Spielberger, C. D. (1989). Manual for the Dutch Version of the State-Trait Anxiety Inventory for Children (STAIC). Lisse: Swets \& Zeitlinger.

Butwicka, A., Fendler, W., Zalepa, A., Szadkowska, A., Zawodniak-Szalapska, M., Gmitrowicz, A., \& Mlynarski, W. (2016). Psychiatric Disorders and Health-Related Quality of Life in Children With Type 1 Diabetes Mellitus. Psychosomatics, 57(2), 185-193.

Cameron, L. D., Young, M. J., \& Wiebe, D. J. (2007). Maternal trait anxiety and diabetes control in adolescents with type 1 diabetes. Journal of Pediatric Psychology, 32(7), 733-744.

Chow, E. T., Otis, J. D., \& Simons, L. E. (2016). The Longitudinal Impact of Parent Distress and Behavior on Functional Outcomes Among Youth With Chronic Pain. Journal of Pain, 17(6), 729738.

Cline, G. D., Schwartz, D. D., Axelrad, M. E., \& Anderson, B. (2011). A pilot study of acute stress symptoms in parents and youth following diagnosis of type i diabetes. Journal of Clinical Psychology in Medical Settings, 18(4), 416-422.

Garnefski, N., Kraaij, V., \& Spinhoven, P. (2001). Negative life events, cognitive emotion regulation and emotional problems. Personality and Individual Differences, 30(8), 1311-1327.

Geisbüsch, C.-M., \& Bühren, K. (2015). Essstörungen bei Diabetes mellitus. Monatsschrift Kinderheilkunde, 163(7), 696-700.

Glidden, L. M., Billings, F. J., \& Jobe, B. M. (2006). Personality, coping style and well-being of parents 
rearing children with developmental disabilities. Journal of Intellectual Disability Research, 50(12), 949-962.

Goubert, L., \& Simons, L. E. (2013). Cognitive styles and processes in paediatric pain. Oxford Textbook of Paediatric Pain, 95-101.

Haugstvedt, a, Wentzel-Larsen, T., Graue, M., Søvik, O., \& Rokne, B. (2010). Fear of hypoglycaemia in mothers and fathers of children with Type 1 diabetes is associated with poor glycaemic control and parental emotional distress: a population-based study. Diabetic Medicine : A Journal of the British Diabetic Association, 27(1), 72-8.

Helgeson, V. S., Becker, D., Escobar, O., \& Siminerio, L. (2012). Families with children with diabetes: Implications of parent stress for parent and child health. Journal of Pediatric Psychology, 37(4), 467-478.

Hilliard, M. E., McQuaid, E. L., Nabors, L., \& Hood, K. K. (2014). Resilience in youth and families living with pediatric health and developmental conditions: Introduction to the special issue on resilience. Journal of Pediatric Psychology, 40(9), 835-839.

Hilliard, M. E., Monaghan, M., Cogen, F. R., \& Streisand, R. (2011). Parent stress and child behaviour among young children with type 1 diabetes. Child: Care, Health and Development, 37(2), 224232.

Hoorelbeke, K., Marchetti, I., De Schryver, M., \& Koster, E. H. W. (2016). The interplay between cognitive risk and resilience factors in remitted depression: A network analysis. Journal of Affective Disorders, 195, 96-104.

Jaser, S., Linsky, R., \& Grey, M. (2014). Coping and Psychological Distress in Mothers of Adolescents with Type 1 Diabetes. Maternal and Child Health Journal, 18(1), 101-108.

Jaser, S., Whittemore, R., Ambrosino, J. M., Lindemann, E., \& Grey, M. (2007). Mediators of Depressive Symptoms in Children with Type 1 Diabetes and their Mothers. Journal of Pediatric 
Psychology, 33(5), 509-519.

Kovacs, M. (1981). Rating scales to assess depression in school-aged children. International Journal of Child \& Adolescent Psychiatry, 46(5-6), 305-315.

Kovacs, M., Goldstone, D., Obrosky, D. S., \& Bonare, L. K. (1997). Psychiatric disorders in youths with IDDM: Rates and risk factors. Diabetes Care, 20(1), 36-44.

Kraaij, V., \& Garnefski, N. (2012). Coping and depressive symptoms in adolescents with a chronic medical condition: A search for intervention targets. Journal of Adolescence, 35(6), 1593-1600.

Kraaij, V., \& Garnefski, N. (2015). Cognitive, Behavioral and Goal Adjustment Coping and Depressive Symptoms in Young People with Diabetes: A Search for Intervention Targets for Coping Skills Training. Journal of Clinical Psychology in Medical Settings, 22(1), 45-53.

Lowes, L., Gregory, J. W., \& Lyne, P. (2005). Newly diagnosed childhood diabetes: A psychosocial transition for parents? Journal of Advanced Nursing, 50(3), 253-261.

Mullins, L. L., Feummeler, B. F., Hoff, A., Chaney, J. M., Van Pelt, J., \& Ewing, A. (2004). The Relationship of Parental Overprotection and Perceived Child Vulnerability to Depressive Symptomotology in Children With Type 1 Diabetes Mellitus: The Moderating Influence of Parenting Stress. Children's Health Care, 33, 21-34.

Nieuwesteeg, A. M., Hartman, E. E., Aanstoot, H. J., van Bakel, H. J. A., Emons, W. H. M., van Mil, E., \& Pouwer, F. (2016). The relationship between parenting stress and parent-child interaction with health outcomes in the youngest patients with type 1 diabetes (0-7 years). European Journal of Pediatrics, 175(3), 329-338.

Norizan, A., \& Shamsuddin, K. (2010). Predictors of parenting stress among Malaysian mothers of children with Down syndrome. Journal of Intellectual Disability Research, 54(11), 992-1003.

Patterson, C. C., Gyürüs, E., Rosenbauer, J., Cinek, O., Neu, a., Schober, E., ... Soltész, G. (2012). 
Trends in childhood type 1 diabetes incidence in Europe during 1989-2008: Evidence of nonuniformity over time in rates of increase. Diabetologia, 55(8), 2142-2147.

Patton, S. R., Dolan, L. M., Henry, R., \& Powers, S. W. (2008). Fear of hypoglycemia in parents of young children with type 1 diabetes mellitus. Journal of Clinical Psychology in Medical Settings, 15(3), 252-259.

Patton, S. R., Dolan, L. M., Smith, L. B., Thomas, I. H., \& Powers, S. W. (2011). Pediatric Parenting Stress and Its Relation to Depressive Symptoms and Fear of Hypoglycemia in Parents of Young Children with Type 1 Diabetes Mellitus. Journal of Clinical Psychology in Medical Settings, 18(4), $345-352$.

Roelofs, J., Braet, C., Rood, L., Timbremont, B., van Vlierberghe, L., Goossens, L., \& van Breukelen, G. (2010). Norms and screening utility of the Dutch version of the Children's Depression Inventory in clinical and nonclinical youths. Psychol Assessment, 22(4), 866-877.

Sieberg, C. B., Williams, S., \& Simons, L. E. (2011). Do parent protective responses mediate the relation between parent distress and child functional disability among children with chronic pain? Journal of Pediatric Psychology, 36(9), 1043-1051.

Spielberger, C. D., Edwards, C. D., Lushene, R. E., Montuori, J., \& Platzek, D. (1973). The state-trait anxiety inventory for children (preliminary manual). Palo Alto, CA: Consluting Psychologists Press.

Spinhoven, P., Ormel, J., Sloekers, P. P., Kempen, G. I., Speckens, a E., \& Van Hemert, a M. (1997). A validation study of the Hospital Anxiety and Depression Scale (HADS) in different groups of Dutch subjects. Psychological Medicine, 27(2), 363-370.

Streisand, R., Mackey, E. R., Elliot, B. M., Mednick, L., Slaughter, I. M., Turek, J., \& Austin, A. (2008). Parental anxiety and depression associated with caring for a child newly diagnosed with type 1 diabetes: Opportunities for education and counseling. Patient Education and Counseling, 73(2), 
333-338.

Streisand, R., Swift, E., Wickmark, T., Chen, R., \& Holmes, C. S. (2005). Pediatric Parenting Stress Among Parents of Children with Type 1 Diabetes: The Role of Self-Efficacy, Responsibility, and Fear. Journal of Pediatric Psychology, 30(6), 513-521.

van der Veek, S. M., Kraaij, V., \& Garnefski, N. (2009). Cognitive coping strategies and stress in parents of children with Down syndrome: a prospective study. Intellectual \& Developmental Disabilities, 47(4), 295-306.

Vanderhasselt, M. A., Koster, E. H. W., Onraedt, T., Bruyneel, L., Goubert, L., \& De Raedt, R. (2014). Adaptive cognitive emotion regulation moderates the relationship between dysfunctional attitudes and depressive symptoms during a stressful life period: A prospective study. Journal of Behavior Therapy and Experimental Psychiatry, 45(2), 291-296.

Vervoort, T., Goubert, L., Vandenbossche, H., Van Aken, S., Matthys, D., \& Crombez, G. (2011). Child's and parent's catastrophizing about pain is associated with procedural fear in children: a study in children with diabetes and their mothers. Psychological Reports.

Vrijmoet-Wiersma, C. M. J., Hoekstra-Weebers, J. E. H. M., De Peinder, W. M. G. M., Koopman, H. M., Tissing, W. J. E., Treffers, P. D. A., ... Egeler, R. M. (2010). Psychometric qualities of the Dutch version of the Pediatric Inventory for Parents (PIP): A multi-center study. Psycho-Oncology, 19(4), 368-375.

Whittemore, R. (2012). Psychological Experience of Parents of Children With Type 1 Diabetes: A Systematic Mixed-Studies Review. Diabetes Educ., 38(4), 562-579.

Williams, L. B., Laffel, L. M. B., \& Hood, K. K. (2009). Diabetes-specific family conflict and psychological distress in paediatric Type 1 diabetes. Diabetic Medicine, 26(9), 908-914.

Zigmond, A. S., \& Snaith, R. P. (1983). The Hospital Anxiety and Depression Scale. Acta Psychiatrica Scandinavica, 67(6), 361-370. 
Table 1. Demographic characteristics of the sample.

\begin{tabular}{llll}
\hline & Mean & Range & SD \\
\hline Child age & 12 & $8-15$ & 2.07 \\
Time since diagnosis & 56 & $1-145$ & 39.80 \\
Mother age & 41 & $29-56$ & 4.98 \\
Mother education & $53.5 \%<$ Highly educated (beyond 18 years), $33 \%<$ High school, $14 \%<$ middle school \\
Marital status & $76.7 \%$ married/cohabiting, $14 \%$ divorced, $4.7 \%$ single, $4.7 \%$ blended family \\
Child gender ${ }^{\text {a }}$ & $58 \%$ Female & \\
\hline Note. Child age (years), time since diagnosis (months). \\
$N=43$ \\
${ }^{a}$ gender: $0=$ boy, $1=$ girl.
\end{tabular}


Table 2. Descriptive statistics and Pearson correlations among maternal distress, cognitive emotion regulation, child outcomes, and child demographics.

\begin{tabular}{|c|c|c|c|c|c|c|c|c|c|}
\hline & \multicolumn{7}{|c|}{ Pearson's correlation coefficients } & \multirow[b]{2}{*}{ Mean } & \multirow[b]{2}{*}{ SD } \\
\hline & 2 & 3 & 4 & 5 & 6 & 7 & 8 & & \\
\hline 1. HADS - mother & $.72 * * *$ & .27 & $.30^{*}$ & -.11 & -.03 & -.05 & .06 & 12.58 & 7.62 \\
\hline 2. PIP - mother & & .20 & $.35^{*}$ & .05 & -.26 & -.28 & -.01 & 95.56 & 27.22 \\
\hline 3. STAIC-trait - child & & & $.66^{* * *}$ & .01 & .03 & $.39 *$ & .06 & 10.95 & 5.67 \\
\hline 4. CDI - child & & & & .05 & .10 & -.02 & .10 & 5.91 & 3.49 \\
\hline 5. CERQ adaptive - mother & & & & & .11 & -.03 & .15 & 12.46 & 2.44 \\
\hline 6. Child age & & & & & & -.03 & .29 & 12 & 2.07 \\
\hline 7. Child gender ${ }^{a}$ & & & & & & & .02 & & \\
\hline 8. Time since diagnosis & & & & & & & --- & & \\
\hline
\end{tabular}

Note. HADS, Hospital Anxiety and Depression Scale; PIP, Pediatric Inventory for Parents; STAIC-trait, State-

Trait Anxiety Inventory for Children; CDI, Child Depression Inventory; CERQ, Cognitive Emotion Regulation

Questionnaire, time since diagnosis (months).

${ }^{\mathrm{a}}$ gender: $0=$ boy, $1=$ girl.

$N=43$

$* \mathrm{p}<.05$ (2-tailed)

$* * \mathrm{p} \leq .01$ (2-tailed)

$* * * \mathrm{p}<.001$ (2-tailed) 
Table 3. Final multiple regression models of maternal distress explaining child anxiety and depression (method backwards).

\begin{tabular}{ccccccc}
\hline Dependent variable & & Model fit & $B$ & $S E$ & $\beta$ & ES \\
& $F$ & $R^{2}$ & & & \\
\hline Child anxiety & $6.77^{* *}$ & .25 & & & & \\
Gender ${ }^{\mathrm{a}}-$ child & & & 5.46 & 1.62 & $.48^{* *}$ & .47 \\
PIP - mother & & 1.88 & .81 & $.33^{*}$ & .35 \\
Child depression & $5.62^{*}$ & .12 & & & & \\
PIP - mother & & & 1.21 & .51 & $.35^{*}$ & .35 \\
\hline
\end{tabular}

Note. $\mathrm{B}=$ unstandardized regression coefficient; $\beta=$ standardized regression coefficient; $\mathrm{ES}=$ effect size $\mathrm{r} ; \mathrm{PIP}$,

Pediatric Inventory for Parents.

${ }^{\text {a } g e n d e r: ~} 0=$ boy, $1=$ girl.

$N=43$

$* \mathrm{p}<.05$

$* * \mathrm{p} \leq .01$ 